\title{
The impact of technology on the study of equine upper airway surgery
}

\author{
John A. Stick and Frederik J. Derksen \\ Department of Large Animal Clinical Sciences, Michigan State University, East Lansing, MI, USA
}

\begin{abstract}
Summary
The objective of this review is to emphasize the importance that technology has played in the diagnosis and choice of surgical treatment of upper airway disorders. We have reviewed the history of the technological advances that have allowed a progression from clinical opinion in the 1970s to objective and accurate determination of upper airway obstructions and their most appropriate surgical correction by the turn of this century. As a result, we have drawn conclusions as to the most effective surgical procedures that should be considered when treating a horse for performance limiting conditions as well as when the treatment is aimed at reducing noise production. The clinical relevance of these technological advances is that partial arytenoidectomy has become the accepted surgical treatment for correction of arytenoid chondritis and, although there are a myriad of surgical procedures aimed at correcting recurrent laryngeal neuropathy (laryngeal hemiplegia), laryngoplasty and laryngeal reinnervation are considered the most successful surgical procedures for performance correction, while bilateral ventriculocordectomy is most effective in eliminating noise production made by this airway obstruction.
\end{abstract}

Keywords: equine upper airway disorders, flow-volume loops, impedance, airflow, surgery of recurrent laryngeal neuropathy (laryngeal hemiplegia), airway flow mechanics

\section{Die Bedeutung der Technologie für die Erforschung der Chirurgie der oberen Atemwege des Pfredes}

Die vorliegende Review behandelt die Wichtigkeit verschiedener Technologien, welche für eine gute Diagnostik und anschliessende chirurgische Behandlung von Erkrankungen der oberen Luftwege heuzutage unerlässlich sind. Aufgezeigt werden die historische Entwicklung und Fortschritte, welche beginnend in den frühen 70-ger Jahren dazu beigetragen haben von klinischen Meinungen zu kritischen und genauen Diagnosen von Obstruktionen der oberen Atemwegserkrankungen, sowie deren chirurgischen Behandlung im jetzigen, neuen Jahrhundert vorzustossen. Es werden Schlussfolgerungen präsentiert, welche die heute besten und effektivsten Methoden zur Behandlung von Pferden mit Leistungseinschränkungen oder zur Reduktion von Atemgeräuschen darstellen. Die klinische Relevanz besteht darin, dass eine partielle Arytenoidektomie als akzeptierte Technik für die Behandlung der Chondritis der Aryknorpel betrachtet wird. Gleichzeitig zeigen sich die Laryngoplastie und laryngeale Innervation als die erfolgversprechendste Methode zur Behebung der Leistungseinschränkungen, und die bilaterale Ventriculo-Kordektomie zur Elimanation von Atemgeräuschen, welche durch Atemwegsobstruktion bedingt sind.

Schlüsselwörter: Erkrankungen der oberen Atemwege, Atemvolumen, Impedanz, Lufffluss, Chirurgie, Kehlkopfpfeifen, Hemiplegia laryngis, Atemluftmechanik

\section{History of diagnosis of upper airway disorders}

Before the fiberoptic endoscope became available in the early 1970s, the upper airway could not be easily examined. At that time the most advanced instrument used to evaluate the upper airway was the rigid endoscope (Fig 1), which only allowed a limited view of a small portion of the upper airway (i.e., the larynx), and was dangerous for the horse and the operator. Only the most common and obvious lesion of recurrent laryngeal neuropathy (RLN) had been described (Fig 2) (Cook 1970). The availability of the fiberoptic endoscope opened up an exciting new era, and descriptions of a variety of upper airway lesions, including lymphoid hyperplasia, entrapment of the epiglottis (Fig 3), and dorsal displacement of the soft palate (DDSP), followed (Aver et al. 1985, Boles et al. 1978, Haynes 1981, Raphel 1982). The source of bleeding in exercise-induced pulmonary hemorrhage (EIPH) was also elucidated by use of the fiberoptic endoscope. While the relationship between strenuous exercise and bleeding in horses had been recognized since at least the early 18 th century, the source of this blood remained a mystery until the 20th century, when Cook used a rigid endoscope to inspect the nasal airways as a possible source of the bleeding (Cook
1974). Because he did not find the source of blood in the nose, the author hypothesized that epistaxis following exercise originated in the lung (Cook 1974). By 1981, the flexible fiberoptic endoscope had become available and, using this new technology, Pascoe et al. reported that, after racing, blood was present in the trachea of $44 \%$ of Thoroughbreds examined (Pascoe et al. 1981). From this study it was concluded that the bleeding originated within the lungs, and the term "exercise-induced pulmonary hemorrhage" (EIPH) was coined.

\section{Modern Era: Videoendoscopy during Exercise}

Upper airway endoscopy in resting horses has two significant shortcomings. First, the evaluation is subjective and it is often difficult to determine the functional significance of a lesion. For example, in the early 1970s, lymphoid hyperplasia was thought to be an important cause of airway obstruction and exercise intolerance in performance horses. Later it was realized that this pharyngeal lesion probably does not cause significant airway obstruction (Bayly et al. 1984). The second shortcoming of fiberoptic endoscopy in resting horses is that 
the dynamic changes that occur in the upper airway during exercise in response to the large pressure swings cannot be appreciated. The first paper on videoendoscopy of the upper airway in exercising horses appeared in 1989 (Stick and Derksen 1989), and was rapidly followed by others (Morris and Seeherman 1990). Using this technique in normal horses, careful observation reveals that on inhalation airway diameter decreases but increases on exhalation. These observations confirm measurements of upper airway impedance, which showed that during exercise upper airway impedance on inhalation is much higher than on exhalation (Derksen et al. 1986). Also, the functional consequence of lesions can

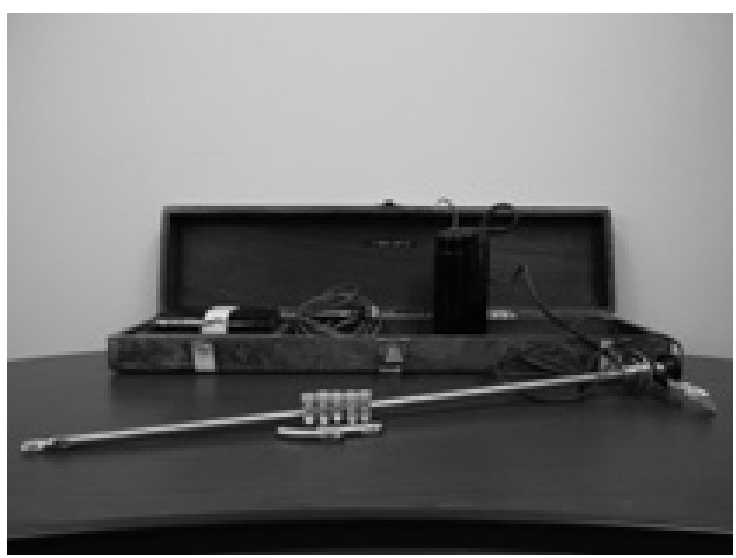

Fig. 1 Photograph of a rigid rhinolaryngoscope that was used to view the equine larynx before the advent of the fiberoptic endoscope.

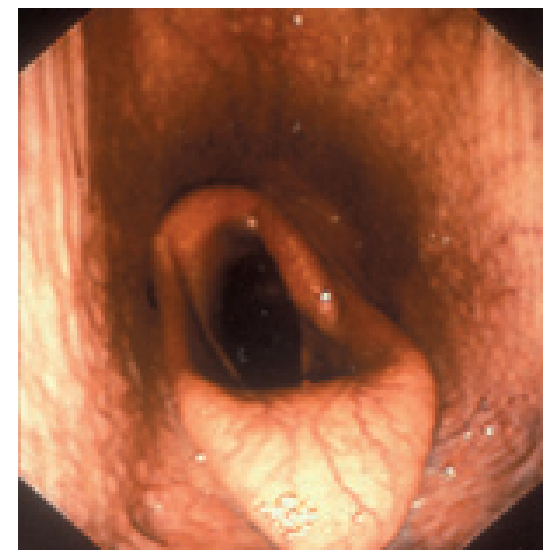

Fig. 2 Appearance of grade 4 recurrent laryngeal neuropathy through a fiberoptic endoscope.

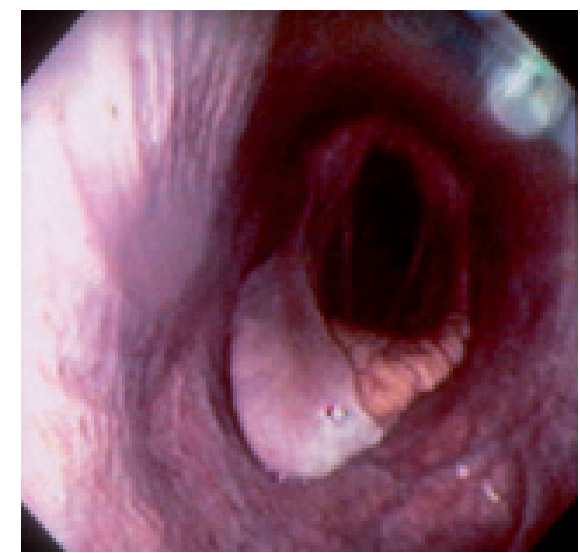

Fig. 3 Endoscopic appearance of epiglottic entrapment in the standing horse. now be determined more easily. Because of the negative intraluminal pressure on inhalation, upper airway lesions tend to move into the lumen during this phase of the respiratory cycle and cause more severe obstructions than might have been suspected from endoscopic evaluations in standing horses (Figs 4 and 5). The functional consequence of upper airway lesions is also affected by the level of fatigue. For example, DDSP may only occur during the final portion of a treadmill evaluation when fatigue is greatest (Fig 6).

For some time, investigators have realized that upper airway endoscopy during treadmill exercise does not always reflect

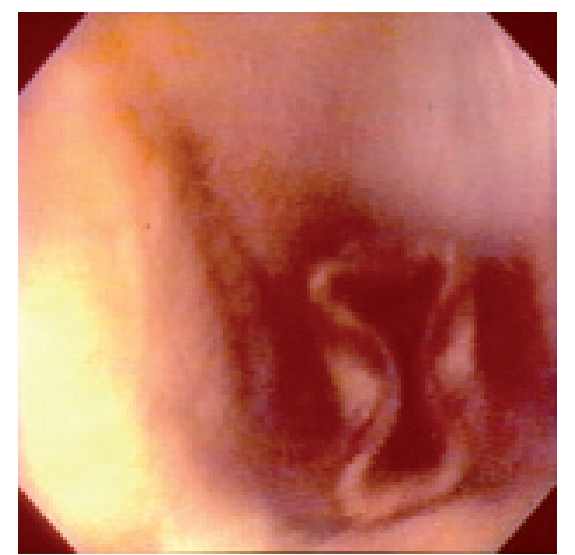

Fig. 4 Photograph of axial deviation of the aryepiglottic folds taken from a videotape of a high-speed treadmill examination.

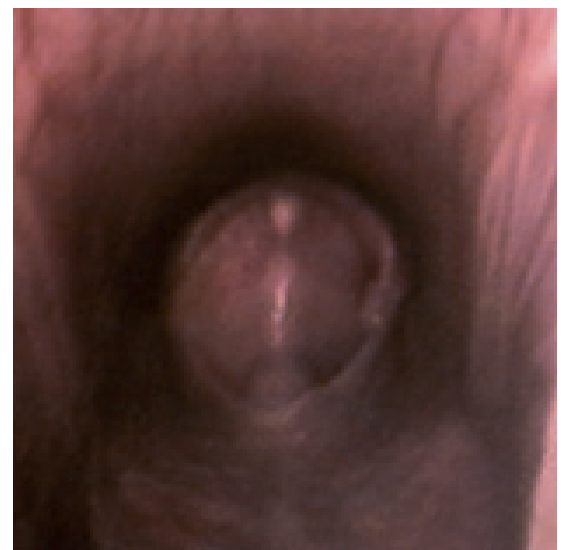

Fig.5 Photograph of epiglottic retroversion taken from a videotape of a high-speed treadmill examination.

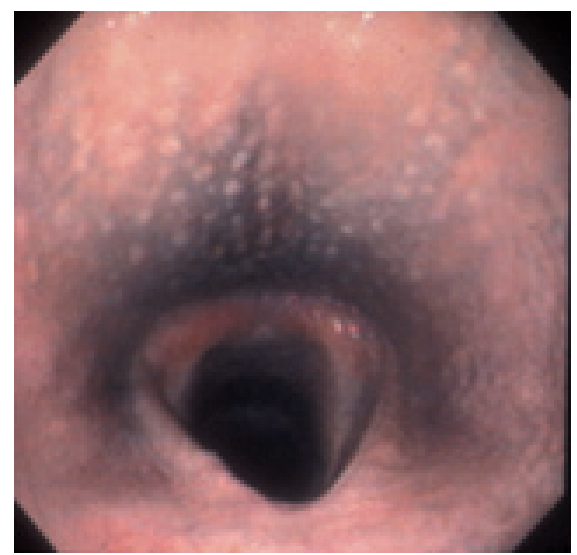

Fig. 6 Endoscopic appearance of dorsal displacement of the soft palate. 
upper airway abnormalities that occur during over the ground exercise (Franklin et al. 2008). For example, horses with a history of upper airway noise during exercise may not make this noise on the treadmill, and suspected DDSP is often not reproducible during treadmill exercise. The reason for these discrepancies is not clear, but may be related to differences in rein tension, head and neck position, and the horse's discomfort with treadmill exercise. Again, advances in technology provided a solution to this diagnostic problem. Telemetric endoscopes are now available that allow endoscopic examination of the upper airway with horses exercising in the field (Pollock et al. 2009). Telemetric endoscopy in exercising horses promises to be a more accurate diagnostic tool in the evaluation of upper airway dysfunction of horses.

\section{Objective measurement of upper airway function: criti- cal examination begins}

Videoendoscopy during exercise, while a valuable diagnostic tool, is still subjective in nature. In some cases, the functional consequences of lesions are readily apparent. In other cases, quantitative evaluation is needed to interpret videoendoscopic findings. The high-speed treadmill has made measurement of upper airway function during exercise practical. Evaluation of airway flow mechanics requires the measurement of air flow and driving pressure. To measure air flow, horses are fitted with a face mask and a flow measurement device. The pressures necessary to move this flow are usually measured in the mid-cervical trachea and at the external nares. The ratio of pressure and flow in a rigid tube is called "resistance." However, when the walls of the tube are not rigid, like in the upper airway, and when air flow rates accelerate and decelerate, as during inhalation and exhalation, the ratio of pressure and flow is best called "impedance" (Shappell et al. 1988). Measurement of air flow mechanics has been used to determine the best surgical treatments for a variety of upper airway obstructive conditions (Fig 7).

If air flow measurements are not available, trans-upper airway pressure during exercise can yield useful information (Williams et al. 1990). As horses begin to exercise, air flows increase in response to increased trans-upper airway pressures. In obstructive diseases, higher driving pressures and lower air flows increase upper airway impedance. Increased trans-upper airway pressures by themselves have been interpreted as evidence of upper airway obstruction. Of course, this interpretation assumes that air flow rates have not decreased, an assumption that is not always correct. Nevertheless, measurement of trans-upper airway pressures, in combination with videoendoscopy, can be helpful in the determination of functional consequences of videoendoscopic findings (Ducharme et al. 1994).

\section{Tidal Breathing Flow-Volume Loops}

Flow-volume loop analysis is a common test of respiratory tract function in human medicine because it is non-invasive, convenient, and sensitive. Air flow rate is continuously plotted against volume during a single, maximal inspiratory and expiratory effort. Initially used in the clinical evaluation of lower airway disease only, flow-volume loop analysis has been used for clinical diagnosis of upper airway obstruction in humans since 1968 (Jordanoglou and Pride 1968). The test requires patient cooperation to perform maximum inhalation and exhalation maneuvers. In doing so, flow rates approach maximum and are associated with significant intraluminal pressure changes, allowing detection of subtle airway obstruction. In veterinary medicine, the non-cooperative nature of our patients has prevented clinical use of maximum flow-volume loop analysis. Clinical evaluation of upper airway function using tidal breathing flow-volume loops (TBFVL) has been attempted in human neonates and infants who are incapable of performing a maximum voluntary respiratory effort on demand (Lodrup Carlsen et al. 1997). When breathing with less than maximum effort, such patients have lower air flow rates that are associated with lack of sensitivity of the TBFVL to detect airway obstruction. Furthermore, the great flow variability associated with TBFVL prevents strict numerical interpretation of loop indices, thus limiting its usefulness in evaluating airway obstruction. Tidal breathing flow-volume loop analysis performed in dogs and horses lacks sensitivity

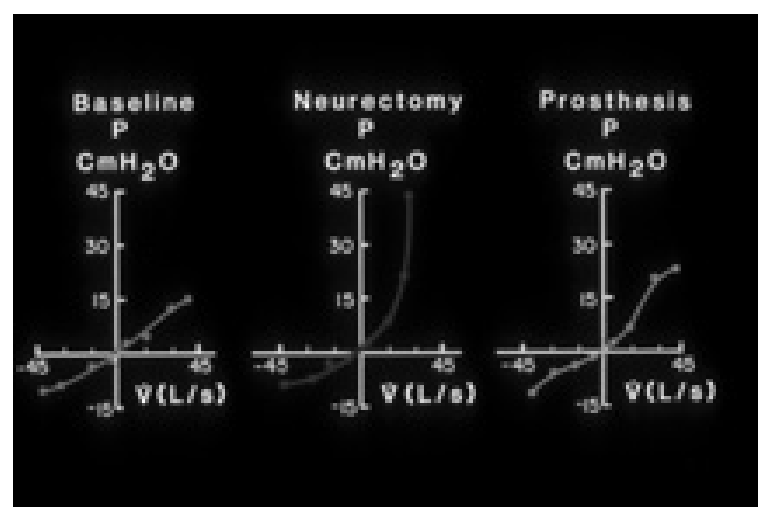

Fig. 7a Graphic illustration of the effects of induced recurrent laryngeal neurectomy followed by laryngoplasty on airflow mechanics. Note that inspiratory pressures increase and airflow is limited by the obstruction (center) compared to return toward normal (left) after laryngoplasty (right).

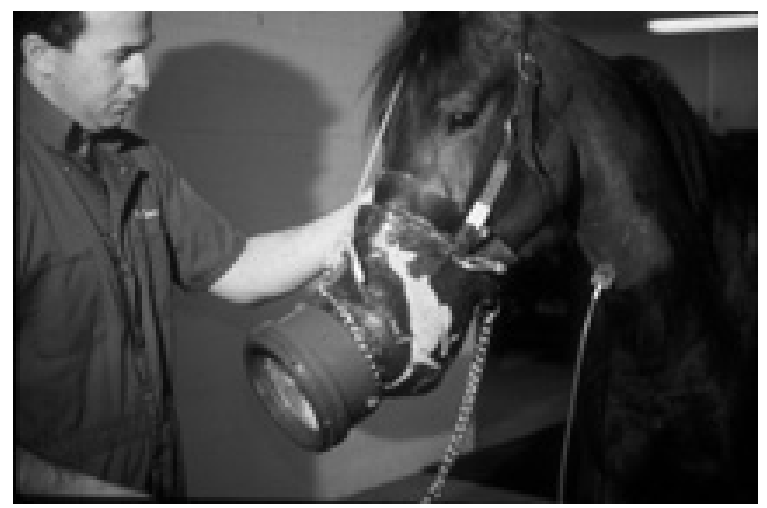

Fig. 7b Pneumotachograph mounted in custom-made facemask used to generate pressure-flow graphs shown in Fig. 7a.

and cannot reliably quantify less than severe obstruction (Amis and Kurpershoek 1986, Petsche et al. 1994). Because the sensitivity of TBFVL in detecting airway obstruction is improved as flow rates increase and, in horses, high-speed treadmill exercise results in near-maximal air flow rates, the use of TBFVL in exercising horses had great potential. In fact these technological advances led to techniques for TBFVL analysis for use in exercising horses and it has been demon- 
strated that the technique is more sensitive than the measurement of flow mechanics in the detection of airway obstruction (Fig 8) (Lumsden et al. 1993, Lumsden et al. 1994).

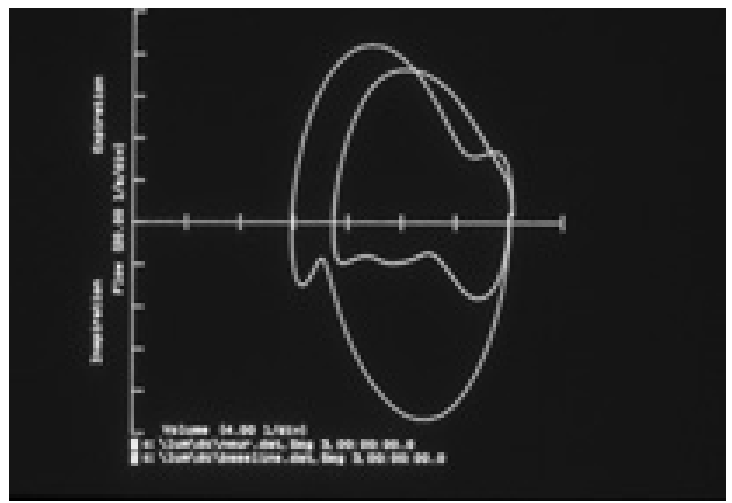

Fig. 8 Tidal breathing flow volume loops showing a normal horse at exercise (large loop) and one with flow limitation (small loop) after induced recurrent laryngeal neuropathy.

\section{Future technological advances in upper airway evalua- tion}

Air flow rates through the relatively narrow upper airway are very high, and the anatomical structure of the upper airway is complex. Therefore it is not surprising that air flow through this structure is equally complex. Computer models are now available to provide new insight into the details of pressure and flow profiles that operate in the upper airway during exercise (Rakesh et al. 2008a). Understanding the details of upper airway flows and pressures during exercise is important because they will provide a better understanding of why certain upper airway regions are more prone to collapse than others. Recently, studies of simulated airflows through computer models of the upper airway provide interesting and sometimes unexpected information (Sridhar et al. 2009). For example, airflow contours and routing through the upper airway during inhalation differ from those during exhalation. Also, there are areas of high turbulence, reverse flows, flow eddies, and great shear stress on certain areas of the upper airway wall (Rakesh et al. 2008a, Sridhar et al. 2009). As computer models become more advanced, our understanding of upper airway flow complexities is likely to clarify why certain regions of the upper airway are predisposed to collapse. In the future it may be possible that real-time measurements of flow contours and pressures can be made in individual exercising horses, providing "individualized medicine" solutions for our patients.

\section{Alleviating airway obstruction in horses with RLN: tech- nology changed surgeries}

In exercising horses with RLN there is dynamic collapse of the affected arytenoid cartilage during inspiration and inspiratory upper airway obstruction (Derksen et al. 1986). The severity of dynamic collapse and upper airway obstruction depends on the degree of laryngeal paresis (Martin et al. 2000). The obstruction is characterized by increased inspiratory transupper airway pressure, inspiratory impedance and inspiratory time, and decreased inspiratory peak flows, respiratory fre- quency, and minute ventilation. Hypoxemia and hypercapnia are also observed (Derksen et al. 1986). Treatments for laryngeal hemiplegia include prosthetic laryngoplasty, ventriculectomy, ventriculocordectomy, laser cordectomy, partial, total, and subtotal arytenoidectomy, and laryngeal reinnervation.

\section{Prosthetic Laryngoplasty}

The prosthetic laryngoplasty technique was first described by Marks in 1970 (Marks et al. 1970). The goal of the procedure is to produce mechanical abduction of the arytenoid cartilage in a position near to full abduction (Fig 9). Laryngoplasty returns upper airway flow mechanics to baseline levels already 30 days after surgery (Derksen et al. 1986, Shappell et al. 1988). Stabilization of the affected arytenoid is more important than the degree of abduction. Indeed, there is no correlation between the degree of arytenoid abduction and residual airway obstruction following surgery (Russell and Slone 1994). Prosthetic laryngoplasty is presently the technique of choice for the treatment of RLN in horses where airway obstruction and exercise intolerance are the primary concern (Hawkins et al. 1997, Russell and Slone 1994). However, postoperative complications are common and include prosthetic failure, dysphagia, coughing, and infection (Hawkins ef al. 1997).

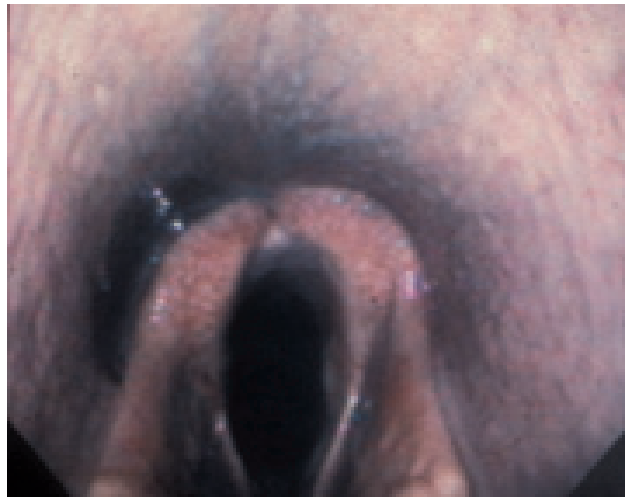

Fig. 9 Endoscopic appearance of the larynx following laryngoplasty demonstrating the optimal position of the left arytenoid.

\section{Ventriculocordectomy}

Ventriculectomy or sacculectomy, refers to the removal of the laryngeal saccule (Fig 10). The objective of ventriculectomy is to produce abduction of the affected arytenoid cartilage by formation of adhesions between the arytenoid and thyroid cartilages and to reduce filling of the ventricle with air during inspiration. Vocal cordectomy is often performed alone or in conjunction with ventriculectomy (in which case it is then referred to as ventriculocordectomy). These procedures may be performed unilaterally or bilaterally and remaining tissues are either left to heal by second intention or are sutured. Variations in surgical techniques are likely to influence efficacy. Unilateral ventriculectomy alone does not improve upper airway function 30 days after surgery (Shappell et al. 1988). Both unilateral vocal cordectomy and bilateral ventriculocordectomy reduce but do not eliminate upper airway obstruction in laryngeal hemiplegia-affected horses (Brown et al. $2003,2005)$. These effects are already evident 30 days after 
surgery. The beneficial effects of prosthetic laryngoplasty on upper airway flow mechanics are not enhanced with bilateral ventriculocordectomy (Tetens et al. 1996).

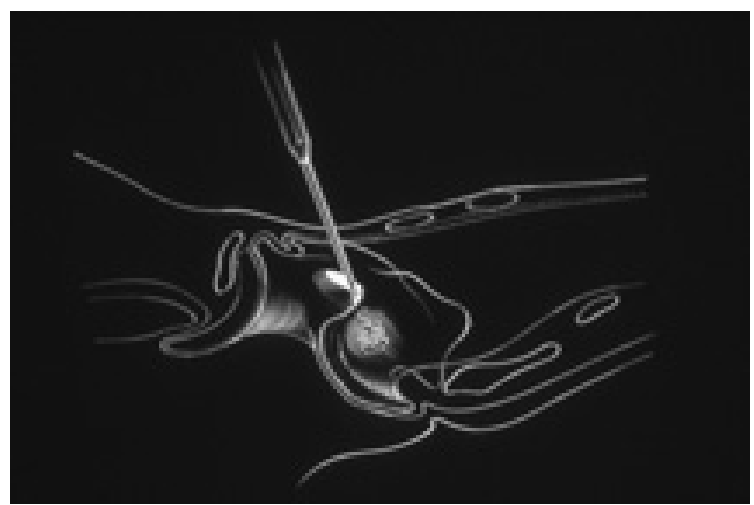

Fig. 10 Schematic representation of the roaring burr placed into the laryngeal saccule through a laryngotomy that is used to evert the ventricle for removal.

\section{Arytenoidectomy}

Arytenoidectomy is rarely used as a first choice surgical technique in horses with RLN. However, following failed prosthe-

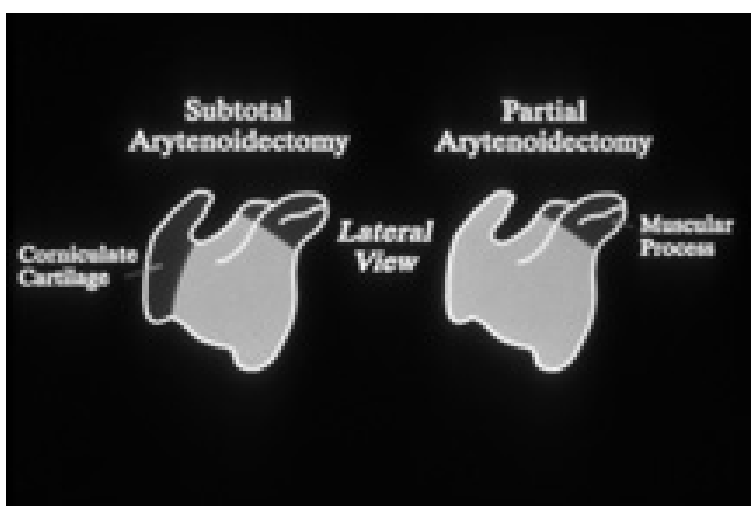

Fig. 11 Drawing demonstrating the difference between a subtotal arytenoidectomy, which spares the corniculate cartilage and muscular process, and a partial arytenoidectomy, which leaves only the muscular process in situ

tic laryngoplasty or in cases of arytenoid chondritis, arytenoidectomy is indicated. When performing the arytenoidectomy procedure, the total arytenoid cartilage may be removed, (total arytenoidectomy), the muscular process may be left in place (partial arytenoidectomy), or the corniculate process may be spared (subtotal arytenoidectomy) (Fig 11).

Partial arytenoidectomy combined with bilateral ventriculocordectomy improves upper airway flow mechanics in exercising horses with experimentally induced laryngeal hemiplegia (Lumsden et al. 1994). However, flow volume loop analysis demonstrates that some airway obstruction remains, and postoperative racing performance is not as good as following prosthetic laryngoplasty combined with ipsilateral ventriculocordectomy (Witte et al. 2009). Complications following arytenoidectomy are common and include dysphagia and coughing. In search of an arytenoidectomy technique with fewer complications, the subtotal arytenoidectomy technique was proposed. However, subtotal arytenoidectomy fails to improve upper airway function (Belknap et al. 1990). Endoscopic evaluation during exercise following subtotal arytenoidectomy demonstrates that the unsupported corniculate process collapses into the airway during inhalation.

\section{Laryngeal Reinnervation}

Because treatments for RLN may be ineffective or associated with serious complications, there has been interest in finding a more physiological way to fix the problem; hence the interest in laryngeal reinnervation. Branches of the first cervical nerve and associated omohyoideus muscle are transplanted into the affected cricoarytenoideus dorsalis muscle (Fig 12). The nerve muscle pedicle graft technique is effective in restoring upper airway flow mechanics in horses with experimentally induced laryngeal hemiplegia (Fulton et al. 1991). Variations in the placement of nerve muscle pedicles are likely to affect efficacy (Cramp et al. 2009a), and multiple grafts have been shown to return horses to racing within 6-8 months (Fulton et al. 2003). Complications associated with this procedure are mild and rare. It has been reported that the nerve muscle pedicle graft technique is as effective as prosthetic laryngoplasty in returning thoroughbred horses to competitive racing (Fulton et al. 2003). Thus, the nerve muscle pedicle

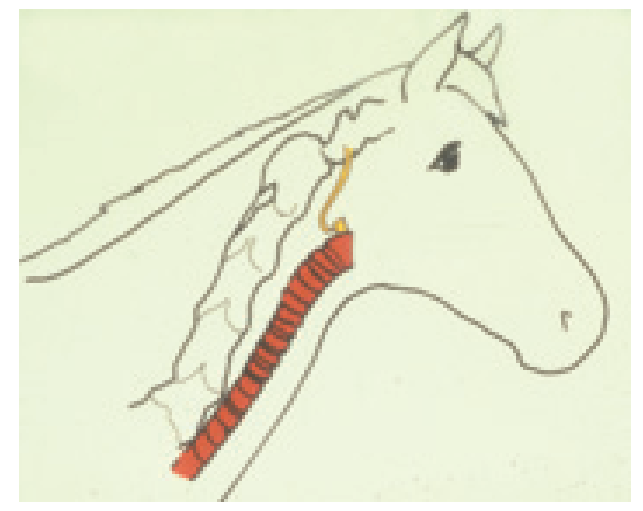

Fig. 12 Drawing demonstrating the position of the neuromuscular pedicle graft after transplantation.

graft technique is recommended when complications associated with other techniques are unacceptable to the owner, and when length of time between surgery and return to athletic activity is less important.

How effective are surgical procedures in restoring athletic performance in RLN-affected racehorses? It was demonstrated that elite Thoroughbred yearlings with grade 3 or 4 RLN had reduced racing performance as adults (Stick et al. 2001). If it can be assumed that these yearlings received the best available care, these data suggest that current surgical treatments cannot fully restored athletic performance in elite racing Thoroughbreds with RLN. This conclusion is supported by another studies (Strand et al. 2000). However, a more recent study suggests that prosthetic laryngoplasty combined with ipsilateral ventriculocordectomy restores racing performance in Thoroughbred horses with grade 3 but not grade 4 RLN (Witte et al. 2009). 


\section{Upper airway sound}

Soundness of wind has been a requirement of athletic horses for centuries. Initially, upper respiratory disease is suspected in an exercising horse when an abnormal respiratory noise is heard and when the horse's performance is reduced. While performance reduction can be caused by dysfunction in many systems, respiratory noise during exercise is specific for an upper airway problem. Without the use of technology, it is difficult to evaluate upper airway noise in terms of pitch, loudness, or phase of the respiratory cycle. Even the jockey or driver, who is nearest to the source of the noise most of the time, does not hear respiratory sounds well because the noise is obscured by other sounds associated with exercise such as wind noise and foot fall. A listener standing near the track gets a snapshot at best. Therefore, techniques have been developed to record and analyze respiratory sounds in exercising horses (Derksen et al. 2001, Franklin et al. 2003)(Fig.13). Respiratory sounds can be recorded under

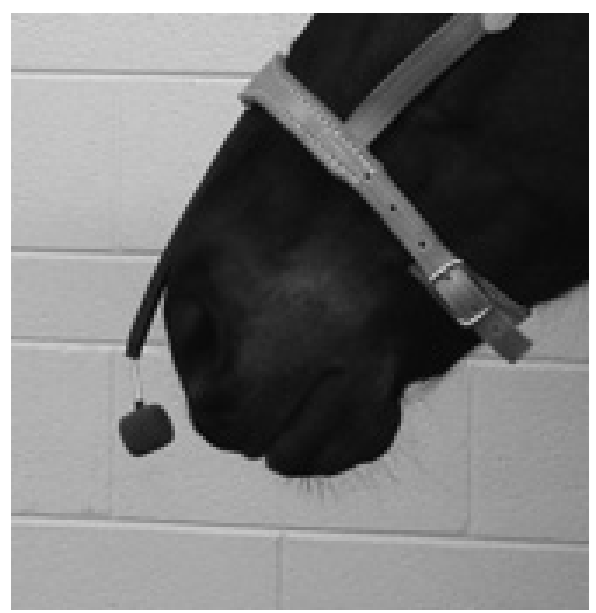

Fig. 13 Photograph of a unidirectional microphone used to record upper airway noise which can be used in spectrum analysis to determine noise intensity and frequency in each phase of the respiratory cycle.

field conditions with the horse exercising in its natural environment and analyzed later using spectrum analysis. Endoscopy and respiratory sound analysis are complementary, each helping to better interpreted findings of the other. For sport horses, respiratory noise caused by conditions such as
RLN can be more important than the obstruction itself. There is now information in the literature describing not only the efficacy of surgical procedures in improving upper airway flow mechanics in exercising horse with RLN, but also the best technique for noise reduction (see below). This information is also important for race horses, as residual respiratory noise after surgery is often interpreted as failure to improve upper airway flow mechanics, and this correlation has been shown to be incorrect.

\section{Alleviating upper airway noise in norses with RLN}

When selecting from the many surgical options for treating RLN, veterinary surgeons must weigh the ability of the surgical procedure to improve upper airway function and/or reduce the associated respiratory noise against the likelihood and severity of postoperative complications. Thus, the surgical procedures selected will vary depending on the intended use of the horse after surgery, the expectations of its owners regarding noise reduction, and the risk tolerance of the owner for postoperative complications (Table 1).

The source of the RLN-associated noise is not clearly understood. Therefore, the efficacy of surgical procedures to reduce the noise is difficult to predict. Several recent studies have addressed this issue. In one study, bilateral ventriculocordectomy effectively reduced respiratory noise in horses with experimentally induced laryngeal hemiplegia (Brown et al. 2003). Respiratory noises associated with the condition were almost completely eliminated, although spectrum analysis revealed some residual abnormal respiratory sounds. Up to 90 days were required before the effect of surgery on noise reduction was optimal.

In recent years, endoscopically guided laser surgery has become popular in the upper airway. The advantage of this technique is that general anesthesia is not required, thus reducing the risk to the horse and the cost to the owner. We have evaluated unilateral laser vocal cordectomy, and concluded that the procedure does not effectively reduce upper airway noise in horses with experimentally induced laryngeal hemiplegia (Brown et al. 2005). Unilateral laser cordectomy results in removal of the left vocal cord, but not the laryngeal ventricle. This study suggests therefore that the laryngeal ventricle rather than the vocal cord may be the

Table 1 Effectiveness of different surgical procedures on performance and noise reduction

\begin{tabular}{lcc}
\hline Procedure & Improved performance & Noise reduction \\
\hline Laryngoplasty & +++ & + \\
Laryngeal reinnervation & +++ & $\mathrm{n} / \mathrm{a}$ \\
Partial arytenoidectomy & ++ & $\mathrm{n} / \mathrm{a}$ \\
Subtotal arytenoidectomy & 0 & $\mathrm{n} / \mathrm{a}$ \\
Unilateral vocal cordectomy & + & 0 \\
Unilateral ventriculectomy & 0 & $\mathrm{n} / \mathrm{a}$ \\
Unilateral ventriculocordectomy & ++ & + \\
Bilateral ventriculectomy & ++ & ++ \\
Bilateral ventriculocordectomy & ++ & +++ \\
\hline
\end{tabular}

Key: $+++=$ optimally effective, $++=$ somewhat effective, $+=$ poorly effective, $0=$ not effective, $n / a=$ insufficient data available 
source of LH-associated noise. This notion was confirmed by a subsequent study of laser-assisted unilateral ventriculocordectomy, which demonstrated that the procedure decreases inspiratory noise, but not as effectively as bilateral ventriculocordectomy (Robinson et al. 2006). Therefore, it is our recommendation that both ventricles and both vocal cords be removed when horses are being treated to reduce noise production.

The most commonly used surgical technique to treat RLN is prosthetic laryngoplasty, because this procedure returns upper airway flow mechanics to baseline values (Derksen et al. 1986). Also, the success of this surgery for returning horses to racing has been well documented (Witte et al. 2009). Prosthetic laryngoplasty reduces upper airway noise in horses with laryngeal hemiplegia, but is not as effective as bilateral ventriculocordectomy in this regard (Brown et al. 2004). However, respiratory noise reduction occurs more rapidly than with bilateral ventriculocordectomy.

Reduction of respiratory noise "as discerned by the critical ear" is often used to determine the surgical success of prosthetic laryngoplasty (Marks et al. 1970). This method of evaluation is distinctly subjective in nature, and assumes a tight correlation between respiratory noise and airway obstruction. In fact, in horses with experimentally induced laryngeal hemiplegia, the correlation between residual airway obstruction following prosthetic laryngoplasty and residual noise is weak (Brown et al. 2004). Therefore, the residual noise during exercise cannot be used as a predictor of improvement in upper airway function in individual horses following laryngoplasty. As long as the affected arytenoid cartilage is stabilized by the prosthetic laryngoplasty surgery, the degree of arytenoid abduction obtained does not appear to affect upper airway flow mechanics, although other authors have reached different conclusions (Rakesh et al. 2008b). Interestingly, in one study it was found that the greater the arytenoid abduction, the louder the residual respiratory noise (Brown et al. 2004). Explanation of this observation will require a better understanding of the source of the respiratory noise.

\section{Draft Horses with RLN}

The treatment of RLN in draft horses warrants special considerations. Draft horses, often weighing more than $900 \mathrm{~kg}$, have greater anesthetic risks then light horses including prolonged recovery, myopathy, peripheral nerve injury and death (Kraus et al. 2003). Therefore, in our clinic we prefer to avoid surgical techniques such as prosthetic laryngoplasty that require general anesthesia. Instead unilateral or bilateral vocal cordectomy, ventriculectomy, or ventriculocordectomy are performed with the horses standing under sedation. Until recently, little was known about the efficacy of ventriculectomy or ventriculocordectomy in draft horses, although a single study of draft horse owner satisfaction suggests that RLN can be treated successfully by unilateral ventriculectomy without prosthetic laryngoplasty (Bohanon et al. 1990). We found that both bilateral ventriculectomy and ventriculocordectomy significantly reduce upper airway noise but that ventriculocordectomy was more effective than ventriculectomy (Cramp et al. 2009b). Furthermore, the procedure had few postoperati- ve complications. For these reasons we recommend bilateral ventriculocordectomy as the preferred treatment for RLN in draft horses.

\section{References}

Amis T. C. and Kurpershoek C. (1986) Tidal breathing flow-volume loop analysis for clinical assessment of airway obstruction in conscious dogs. Am. J. Vet. Res. 47, 1002-1006

Aver D. E., Wilson R. G. and Groenendyk S. (1985) Pharyngeal lymphoid hyperplasia in Thoroughbred racehorses in training. Aust. Vet. J. 62, 124-126

Bayly W. M., Grant B. D. and Breeze R. G. (1984) Arterial blood gas tension and acid base balance during exercise in horses with pharyngeal lymphoid hyperplasia. Equine Vet. J. 16, 435-438

Belknap J. K., Derksen F. J., Nickels F. A., Stick J. A. and Robinson N. E. (1990) Failure of subtotal arytenoidectomy to improve upper airway flow mechanics in exercising standardbreds with induced laryngeal hemiplegia. Am. J. Vet. Res. 51, 1481-1487

Bohanon T. C., Beard W. L. and Robertson J. T. (1990) Laryngeal hemiplegia in draft horses. A review of 27 cases. Vet. Surg. 19, 456-459

Boles C. L., Raker C. W. and Wheat J. F. (1978) Epiglottic entrapment by arytenoepiglottic folds in the horse. J. Am. Vet. Med. Assoc. 172, 338-342.

Brown J. A., Derksen F. J., Stick J. A., Hartmann W. M. and Robinson N. E. (2003) Ventriculocordectomy reduces respiratory noise in horses with laryngeal hemiplegia. Equine Vet. J. 35, 570-574

Brown J. A., Derksen F. J., Stick J. A., Hartmann W. M. and Robinson N. E. (2004) Effect of laryngoplasty on respiratory noise reduction in horses with laryngeal hemiplegia. Equine Vet. J. 36, 420-425.

Brown J. A., Derksen F. J., Stick J. A., Hartmann W. M. and Robinson N. E. (2005) Laser vocal cordectomy fails to effectively reduce respiratory noise in horses with laryngeal hemiplegia. Vet. Surg. 34, 247-252

Cook W. R. (1970) A comparison of idiopathic laryngeal paralysis in man and horse. Laryngol. Otolaryngol. 84, 819-835

Cook W. R. (1974) Epistaxis in the racehorse. Equine Vet J 6, 45-58.

Cramp P., Derksen F. J., Stick J. A., de Feijter-Rupp H., Elvin N. G., Hauptman J. and Robinson N. E. (2009a) Effect of magnitude and direction of force on laryngeal abduction: implications for the nerve-muscle pedicle graft technique. Equine Vet. J. 41, 328-333

Cramp P., Derksen F. J., Stick J. A., Nickels F. A., Brown K. E., Robinson P. and Robinson N. E. (2009b) Effect of ventriculectomy versus ventriculocordectomy on upper airway noise in draught horses with recurrent laryngeal neuropathy. Equine Vet. J. $41,729-734$

Derksen F. J., Holcombe S. J., Hartmann W., Robinson N. E. and Stick J. A. (2001) Spectrum analysis of respiratory sounds in exercising horses with experimentally induced laryngeal hemiplegia or dorsal displacement of the soft palate. Am. J. Vet. Res. 62, 659-664

Derksen F. J., Stick J. A., Scott E. A., Robinson N. E. and Slocombe R. $F$. (1986) Effect of laryngeal hemiplegia and laryngoplasty on airway flow mechanics in exercising horses. Am. J. Vet. Res. 47, 16-20

Ducharme N. G., Hackett R. P., Ainsworth D. M., Erb H. N. and Shannon K. J. (1994) Repeatability and normal values for measurement of pharyngeal and tracheal pressures in exercising horses. Am. J. Vet. Res. 55, 368-374

Franklin H., Burnt J. F. and Allen K. J. (2008) Clinical trials using a telemetric endoscope for use during over-ground exercise: a preliminary study. Equine Vet. J. 40, 712-715

Franklin S. H., Usmar S. G., Lane J. G., Shuttleworth J. and Burn J. F. (2003) Spectral analysis of respiratory noise in horses with upper airway disorders. Equine Vet. J. 35, 264-268

Fulton I. C., Derksen F. J., Stick J. A., Robinson N. E. and Walshaw R. (1991) Treatment of left laryngeal hemiplegia in standardbreds, using a nerve muscle pedicle graft. Am. J. Vet. Res. 52, 1461-1467

Fulton I. C., Stick J. A. and Derksen F. J. (2003) Laryngeal reinnervation in the horse. Vet. Clin. North Am. Equine Pract. 19, 189-208 
Hawkins J. F., Tulleners E. P., Ross M. W., Evans L. H. and Raker C. W. (1997) Laryngoplasty with or without ventriculectomy for treatment of left laryngeal hemiplegia in 230 racehorses. Vet. Surg. 26, 484-491

Haynes P. F. (1981) Persistent dorsal displacement of the soft palate associated with epiglottic shortening in two horses. J. Am. Vet. Med. Assoc. 179, 677-681

Jordanoglou J. and Pride N. B. (1968) Factors determining maximum inspiratory flow and maximum expiratory flow of the lung. Thorax 23, 33-37

Kraus B. M., Parente E. J. and Tulleners E. P. (2003) Laryngoplasty with ventriculectomy or ventriculocordectomy in 104 draft horses (1992-2000). Vet. Surg. 32, 530-538

Lodrup Carlsen K. C., Stenzler A. and Carlsen K. H. (1997) Determinants of tidal flow volume loop indices in neonates and children with and without asthma. Pediatr. Pulmonol. 24, 391-396

Lumsden J. M., Derksen F. J., Stick J. A. and Robinson N. E. (1993) Use of flow-volume loops to evaluate upper airway obstruction in exercising standardbreds. Am. J. Vet. Res. 54, 766-775

Lumsden J. M., Derksen F. J., Stick J. A., Robinson N. E. and Nickels F. A. (1994) Evaluation of partial arytenoidectomy as a treatment for equine laryngeal hemiplegia. Equine Vet. J.. 26, 125-129

Marks D., Mackay-Smith M. P., Cushing L. S. and Leslie J. A. (1970) Use of a prosthetic device for surgical correction of laryngeal hemiplegia in horses. J. Am. Vet. Med. Assoc. 157, 157-163

Martin B. B. Jr., Reef V. B., Parente E. J. and Sage A. D. (2000) Causes of poor performance of horses during training, racing, or showing: 348 cases (1992-1996). J. Am. Vet. Med. Assoc. 216, 554-558

Morris E. A. and Seeherman H. J. (1990) Evaluation of upper respiratory tract function during strenuous exercise in racehorses. J. Am. Vet. Med. Assoc. 196, 431-438

Pascoe J. R., Ferraro G. L., Cannon J. H., Arthur R. M. and Wheat J. D. (1981) Exercise-induced pulmonary hemorrhage in racing thoroughbreds: a preliminary study. Am. J. Vet. Res. 42, 703-707

Petsche V. M., Derksen F. J. and Robinson N. E. (1994) Tidal breathing flow-volume loops in horses with recurrent airway obstruction (heaves). Am. J. Vet. Res. 55, 885-891

Pollock P. J., Reardon R. J., Parkin T. D., Johnston M. S., Tate J. and Love S. (2009) Dynamic respiratory endoscopy in 67 Thoroughbred racehorses training under normal ridden exercise conditions. Equine Vet. J. 41, 354-360

Rakesh V., Datta A. K., Ducharme N. G. and Pease A. P. (2008a) Simulation of turbulent airflow using a CT based upper airway model of a racehorse. J. Biomech. Eng. 130, 031011.

Rakesh V., Ducharme N. G., Cheetham J., Datta A. K. and Pease A. $P$. (2008b) Implications of different degrees of arytenoid cartilage abduction on equine upper airway characteristics. Equine Vet. J. 40, 629-635

Raphel C. F. (1982) Endoscopic findings in the upper respiratory tract of 479 horses. J. Am. Vet. Med. Assoc. 181, 470-473
Robinson P., Derksen F. J., Stick J. A., Sullins K. E., DeTolve P. G. and Robinson N. E. (2006) Effects of unilateral laser-assisted ventriculocordectomy in horses with laryngeal hemiplegia. Equine Vet. J. 38, 491-496

Russell A. P. and Slone D. E. (1994) Performance analysis after prosthetic laryngoplasty and bilateral ventriculectomy for laryngeal hemiplegia in horses: 70 cases (1986-1991). J. Am. Vet. Med. Assoc. 204, 1235-1241

Shappell K. K., Derksen F. J., Stick J. A. and Robinson N. E. (1988) Effects of ventriculectomy, prosthetic laryngoplasty, and exercise on upper airway function in horses with induced left laryngeal hemiplegia. Am. J. Vet. Res. 49, 1760-1765

Sridhar S., Zhuang M., Malinowski R., Derksen F. J. and Robinson N. E. (2009) Using computational fluid dynamics to understand pressure and air flow velocity distributions in the equine upper airway: Pertinence to dorsal displacement of the soft palate. In: World equine airway symposium, Berne, Switzerland. p 242

Stick J. A. and Derksen F. J. (1989) Use of videoendoscopy during exercise for determination of appropriate surgical treatment of laryngeal hemiplegia in a colt. J. Am. Vet. Med. Assoc. 195, 619-622

Stick J. A., Peloso J. G., Morehead J. P., Lloyd J., Eberhart S., Padungtod P. and Derksen F. J. (2001) Endoscopic assessment of airway function as a predictor of racing performance in Thoroughbred yearlings: 427 cases (1997-2000). J. Am. Vet. Med. Assoc. 219, 962-967

Strand E., Martin G. S., Haynes P. F., McClure J. R. and Vice J. D. (2000) Career racing performance in Thoroughbreds treated with prosthetic laryngoplasty for laryngeal neuropathy: 52 cases (1981-1989). J. Am. Vet. Med. Assoc. 217, 1689-1696

Tetens J., Derksen F. J., Stick J. A., Lloyd J. W. and Robinson N. E. (1996) Efficacy of prosthetic laryngoplasty with and without bilateral ventriculocordectomy as treatments for laryngeal hemiplegia in horses. Am. J. Vet. Res. 57, 1668-1673

Williams J. W., Meagher D. M., Pascoe J. R. and Hornof W. J. (1990) Upper airway function during maximal exercise in horses with obstructive upper airway lesions. Effect of surgical treatment. Vet. Surg. 19, 142-147

Witte T. H., Mohammed H. O., Radcliffe C. H., Hackett R. P. and Ducharme N. G. (2009) Racing performance after combined prosthetic laryngoplasty and ipsilateral ventriculocordectomy or partial arytenoidectomy: 135 Thoroughbred racehorses competing at less than $2400 \mathrm{~m}$ (1997-2007). Equine Vet. J. 41, 70-75

John A. Stick, DVM, Dipl ACVS

Department of Large Animal Clinical Sciences

Michigan State University

East Lansing, MI, 48824-1314

USA

stick@cvm.msu.edu 